\title{
On mobility-lifetime products in photorefractive GaAs-AlGaAs quantum wells structures determined by moving grating technique measurements
}

\author{
M. Wichtowski, ${ }^{*}$ E. Weinert-Rączka, E. Miśkiewicz and A. Branecka \\ Faculty of Electrical Engineering, West Pomeranian University of Technology, al Piastów 17, 70-310 Szczecin
}

Received December 05, 2014; accepted December 31, 2014; published December 31, 2014

\begin{abstract}
A method is proposed to determine mobility-lifetime products of photoexcited electrons and holes in semi-insulating GaAsAlGaAs quantum wells structures. The method is based on photoconductivity measurements and optical investigation of photorefractive material response in a wave-mixing setup using the running grating technique.
\end{abstract}

A photorefractive effect (PR) can be employed to extract the values of selected material parameters. The parameter which is frequently determined in PR measurements is the product of carriers mobility and their average lifetime (recombination time). Such experiments are usually carried out in a two-wave mixing setup where two coherent plane waves illuminate a PR crystal and form a sinusoidal interference pattern. Nonuniform illumination induces periodic changes in the refractive index. If the photorefractive grating is spatially shifted with respect to the interference pattern nonreciprocal energy transfer occurs between beams resulting in the enhancement of a signal beam. To increase the phase shift and, consequently, improve the PR gain two methods have been developed: (1) the application of a fast alternating AC external electric field with a stationary interference pattern [1-2], (2) the moving fringe technique in the presence of external an DC electric field [1,2]. In both cases the PR gain $(\Gamma)$ depends on the mobility-lifetime product $(\mu \tau)$ and the spatial period of the grating $(\Lambda)$. Experimentally obtained the $\Gamma$ on $\Lambda$ dependence allows to determine the product $\mu \tau$, treated as a free parameter, by fitting the theoretical curve to measurement data. For conventional PR materials, such as ferroelectrics and sillenites, a theoretical curve is calculated on the basis of standard material equations within the band transport model [1,2], in which material properties are modeled by assuming one deep trap level and one type of carrier, usually electrons.

The described method is not sufficient in the case of photorefractive semiconductors, which often have mixed conductivity (electrons and holes). Moreover in

\footnotetext{
*E-mail: marekw@zut.edu.pl
}

semiconductors such as GaAs and InP under high electric field $(E>4-5 \mathrm{kV} / \mathrm{cm})$ nonlinear transport of hot electrons has to be taken into account. This phenomenon is associated with the intervalley scattering effect leading to the dependence of electron mobility on an electric field i.e. $\mu_{e}=\mu_{e}(E)$ and the appearance of negative differential conductivity (NDC) [2]. The materials having such properties can be exemplified by semi-insulating structures of multiple quantum wells (SI-MQW) GaAsAlGaAs. These materials exhibit strong resonant nonlinearity connected with exciton interband transitions in which electron-hole pairs are generated. Unlike conventional PR materials, the SI-MQW structures for wavelengths corresponding to exciton resonances reveal interband absorption of an order of $(5-10) \times 10^{3} \mathrm{~cm}^{-1}$ and a quadratic electro-optic effect [2]. For this reason SIMQW devices work as thin layers $(\sim 1 \mu \mathrm{m})$ in a transmission geometry with strong electric field applied along well planes. During the writing of interference pattern, a thin diffractive grating is formed on which twowave mixing (TWM) and degenerate four-wave mixing (FWM) occurs simultaneously, observed as higher diffraction orders.

In this work we propose a method of determining mobility-lifetime products for electrons and holes in an SI-MQW sample, based on measurements of an absorption coefficient, electrical conductivity, resonance frequency of a moving grating and recording time of the grating. In part, the results of such measurements were given in the study [3], where TWM and FWM experiments on a $\mathrm{GaAs}-\mathrm{Al}_{0.3} \mathrm{Ga}_{0.7} \mathrm{As} \mathrm{MQW}$ sample with a stationary and running grating were described. In the latter case, the sample was illuminated by two laser beams with the frequency $\omega$ passing through acousto-optical modulators, allowing to obtain a frequency detuning of one of the beams: i.e. $\omega^{\prime}=\omega+\Omega$, where $\Omega \ll \omega$. As a result, the superposition of writing waves of intensities of $I_{1}$ and $I_{2}$ gave the interference pattern: $I(x, t)=\left(I_{1}+I_{2}\right)[1+$ $m \cdot \cos (K x-\Omega t)]$, describing the light pattern with the fringe contrast $m$ moving with the velocity $v=\Omega / K$, where $K=2 \pi / \Lambda$ denotes the grating constant. For a given 
frequency $\Omega=\Omega_{\text {res }}$ one obtains the enhancement of the PR response with the resonant frequency $\Omega_{\text {res }}$ increasing linearly with the average light intensity $\left(I_{0}=I_{1}+I_{2}\right)[3,4]$. In order to obtain theoretical relationships for quantities of interest we refer to the system of material equations given in [2, 5], where both bipolar carrier transport and hot electrons nonlinear transport are included. Analytical solutions of these equations can be found in small signal approximation, i.e. for small fringe contrast $(\mathrm{m} \ll 1)$. Neglecting the diffusion currents for large applied electric fields, one obtains [4]:

(a) resonant detuning frequency $\left(\Omega_{\text {res }}\right)$ :

$$
\Omega_{\mathrm{res}}=A K E_{a}\left(\frac{\left(\mu_{h} \tau_{h}\right)^{2}}{1+\left(\mu_{h} \tau_{h} K E_{a}\right)^{2}}-\frac{\mu_{\text {diff }} \tau_{e} \cdot \mu_{e} \tau_{e}}{1+\left(\mu_{e} \tau_{e} K E_{a}\right)^{2}}\right),
$$

where $A=q(\alpha / h v) I_{0}$ and $\alpha$ is the absorption coefficient, $\tau_{h}, \tau_{e}$ denote average lifetimes for electrons and holes, respectively, $\mu_{h}, \mu_{e}(E), \mu_{d i f f}(E)=\left(d \mu_{e} / d E\right)_{E a}$ denote, consecutively: mobility of holes, mobility of electrons and differential mobility of electrons, which can be negative.

(b) time constant of material response with step-like switching of the writing beams and $\Omega=\Omega_{\text {res }}$,

$$
\tau_{s c}=\frac{1}{A} \cdot \frac{1+\left(\mu_{e} \tau_{e} K E_{a}\right)^{2}}{\mu_{\text {diff }} \tau_{e}+\mu_{h} \tau_{h} \frac{1+\left(\mu_{e} \tau_{e} K E_{a}\right)^{2}}{1+\left(\mu_{h} \tau_{h} K E_{a}\right)^{2}}}
$$

It should be noted, that in the experiments the ratio of writing beam intensities was close to one, what corresponds to high fringe contrast $(m \sim 1)$. To check the validity of Eqs. (1) and (2) we solved material equations numerically by means of the finite difference method for different values of $m$. It turns out that the influence of the fringe contrast on both resonance frequency and response time is small, hence the analytical solutions provide good approximations, as can be seen in Figs. 1a and 1b. In particular, the temporal evolution of space-charge field amplitude can be described by a simple function $E_{\mathrm{sc}}(t)=$ $E_{\mathrm{sc}}{ }^{\max }\left(1-\mathrm{e}^{-t / \tau \mathrm{sc}}\right)$. Note, that in the FWM experiments, the temporal behavior of a diffracted signal is measured instead of a space-charge field itself. For SI-MQW with a quadratic electro-optic effect, the diffraction efficiency, and hence the diffracted signal intensity, are proportional to the square of the amplitude of a refractive index grating, i.e. $I_{\text {diff }}(t) \propto[\Delta n(t)]^{2} \propto\left[E_{0}+E_{\mathrm{sc}}(t)\right]^{4}$. Therefore, the time response of an optical signal is not equal to the space-charge field response time given by Eq. (2).
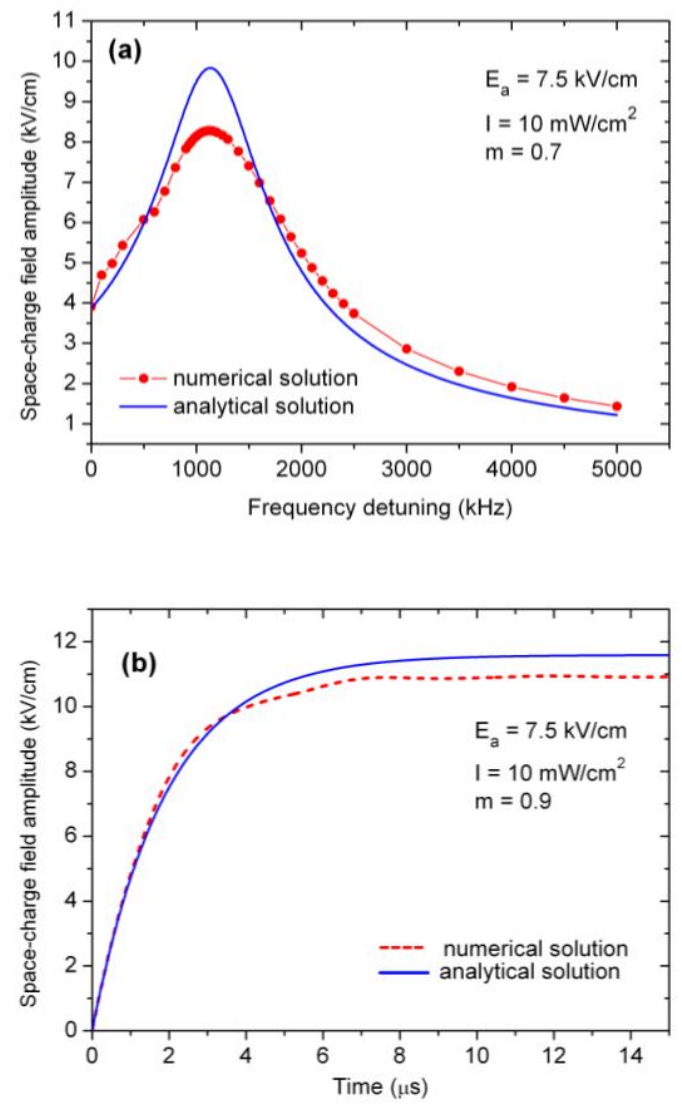

Fig. 1. (a) amplitude of a space-charge field as a function of detuning frequency $\Omega$ calculated numerically and according to Eq. (1) for an applied electric field of $7.5 \mathrm{kV} / \mathrm{cm}$, (b) temporal evolution of the spacecharge field after switching on the light at $t=0$ for resonant detuning frequency.

In addition to the quantities $\Omega_{\mathrm{res}}$ and $\tau_{\mathrm{sc}}$ the values of absorption coefficient for a given wavelength and the density of a current in an illuminated sample are needed:

$$
j=A\left(\mu_{h} \tau_{h}+\mu_{e} \tau_{e}\right) E_{a}
$$

All measurements should be carried out with the same intensity of sample illumination, and without changing the applied voltage. From the above equations, with three free parameters: $\mu_{\mathrm{h}} \tau_{\mathrm{h}}, \mu_{\mathrm{e}} \tau_{\mathrm{e}}$ and $\mu_{\mathrm{diff}} \tau_{\mathrm{e}}$ one can obtain the following equation:

$$
x_{h}^{3}-\left(\frac{j \cdot K}{A}+\Omega_{r e s} \tau_{s c}\right)\left(x_{h}^{2}+1\right)+\left(1+\frac{j \cdot \tau_{s c}}{\varepsilon E_{a}}\right) x_{h}=0,
$$

where $x_{h}=\mu_{h} \tau_{h} K E_{a}$. The equation (4) has one real solution giving the required product $\mu_{\mathrm{h}} \tau_{\mathrm{h}}$ for holes, then the product $\mu_{\mathrm{e}} \tau_{\mathrm{e}}$ for electrons is determined from Eq. (3).

As an example, let us assume the following parameters as in Ref. [3], obtained for wavelength $\lambda=830 \mathrm{~nm}$, average 
intensity of writing beams $I_{0}=3 \mathrm{~mW} / \mathrm{cm}^{2}$, applied field $E_{a}=7.5 \mathrm{kV} / \mathrm{cm}$ and grating period $\Lambda=20 \mu \mathrm{m}$.

The paper [3] contains the measured values of resonant frequency detuning $\Omega_{\mathrm{res}}=70 \mathrm{kHz}$ and time constant of the diffracted signal $\tau_{s c}=15 \mu$ s in the FWM experiment. Taking the absorption coefficient $\alpha=9 \cdot 10^{3}$ $\mathrm{cm}^{-1}$ and the current density $j=26 \mathrm{~mA} / \mathrm{cm}^{2}$ values, which were measured for the similar SI-MQW (GaAs$\mathrm{Al}_{0.3} \mathrm{Ga}_{0.7} \mathrm{As}$ ) sample for an average light intensity of $3 \mathrm{~mW} / \mathrm{cm}^{2}$, one finds from Eq. (4): $\mu_{\mathrm{h}} \tau_{\mathrm{h}} \approx 5 \cdot 10^{-9} \mathrm{~cm}^{2} / \mathrm{V}$ and $\mu_{\mathrm{e}} \tau_{\mathrm{e}} \approx 2 \cdot 10^{-7} \mathrm{~cm}^{2} / \mathrm{V}$. The correctness of determined values of $\mu \tau$ can be verified by performing a few measurements of resonance frequency values for different grating periods, and fitting the theoretical curve $\Omega_{\text {res }}(\Lambda)$ plotted on the basis of Eq. (1) (shown in Fig. 2) to experimental data.

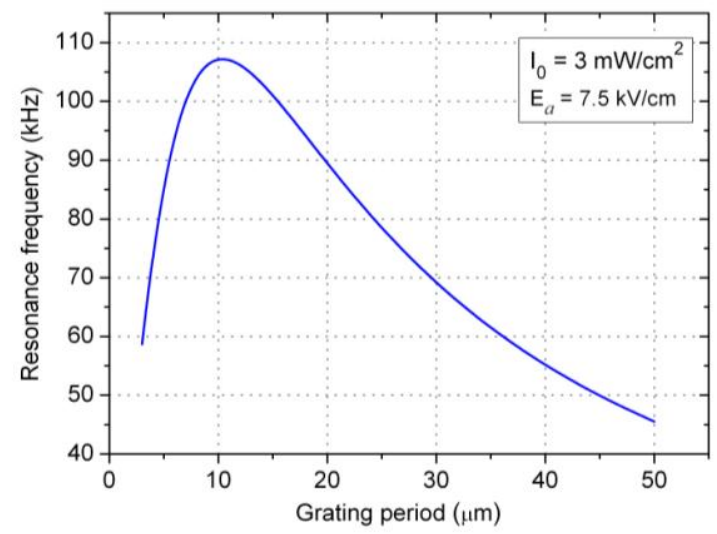

Fig. 2. Theoretical dependence of resonance frequency detuning according to Eq. (1) versus the grating period $(\Lambda)$.

For resonant wavelength $(\sim 830 \mathrm{~nm})$ and moderate external electric field $\left(E_{a} \leq 10 \mathrm{kV} / \mathrm{cm}\right)$, electron-hole pairs are generated in GaAs well layers. Thus, both the current flow and the PR effect occur primarily in gallium arsenide. For GaAs the mobilities of holes and electrons as a function of electric field are fairly well known [6]. This fact permits to evaluate the mean life-time of electrons and holes which are given by $\tau_{\mathrm{e}}=1 /\left(\gamma_{e} N_{e}\right), \tau_{\mathrm{h}}=$ $1 /\left(\gamma_{h} N_{h}\right)$, where $\gamma_{\mathrm{e}}, \gamma_{\mathrm{h}}$ are recombination coefficients and $N_{e}, N_{h}$ denote the trap concentrations for electrons and holes, respectively. At present, the values of recombination coefficients and trap densities can be only estimated with rather rough approximation. This is due to the proton implantation used in semi-insulating MQW process technology $[2,7]$ as well as field-enhanced deeplevel-defect capture-cross-section [8] which can result in the dependence $\gamma=\chi(E)$. A more detailed knowledge of the described parameter values is important in planning the applications of PR semiconductors with mixed conductivity and nonlinear transport, particularly for devices in which the light propagates over longer distances [9].
This work has been partially supported by the National Center for Science under the Project awarded by decision number DEC-2011/01/B/ST7/06234.

\section{References}

[1] L. Solymar, D.J. Webb, A. Grunnet-Jepsen, The physics and applications of photorefractive materials (Clarendon Press: Oxford 1996).

[2] D.D. Nolte, M.R. Melloch, Photorefractive effects and Materials, ed. by D.D.Nolte (Kluwer, Dordrecht, 1995).

[3] S. Balasubramanian, I. Lahiri, Y. Ding, M.R. Melloch, D.D. Nolte, Appl. Phys. B 68, 863 (1999).

[4] M Wichtowski, Appl. Phys. B 115, 505 (2014).

[5] Q. Wang, R.M. Brubaker, D.D. Nolte, J. Opt. Soc. Am. B 9, 1773 (1994).

[6] M. Shur GaAs devices and circuits (Springer, New York, 1989).

[7] D.D. Nolte, J. Appl. Phys. 85, 6259 (1999).

[8] G.C. Valley, H. Rajbenbach, H.J. von Bardeleben, Appl. Phys. Lett. 56, 364 (1989).

[9] A. Ziółkowski J. Opt. 14, 035202 (2012). 